Planetary Science and Astrobiology Decadal Survey 2023-2032

Science White Paper

\title{
Meteoritical Constraints on our Solar System and Beyond
}

\author{
Author: \\ Jasmeet K. Dhaliwal \\ UC, Santa Cruz \\ jkdhaliwal.sio@gmail.com \\ $858-869-4890$
}




\section{Executive Summary}

Meteorites provide important constraints for key stages in planetary evolution, namely metalsilicate differentiation, silicate melting, and impact bombardment. The continued investigation of meteorites, through observational and experimental studies, are important to establishing the unique styles and trajectories of planetary evolution within our solar system. This knowledge can be readily applied to exoplanet science, such as modeling the formation of distant worlds, identifying telescope evidence of volcanism and impact bombardment, and investigating the habitability of Earth-like exoplanets.

\section{Introduction}

Meteorites are valuable materials for investigating important stages in planetary evolution that can influence habitability. These stages include metal-silicate differentiation, silicate melting, and impact bombardment. The planetary conditions during these stages are also important to related processes such as core-formation, magma oceans, and delivery and loss of volatiles. Understanding these aspects of a planet's evolutionary history are important for constraining volcanism styles and volatile inventories, which can ultimately determine whether an atmosphere allows for liquid water.

Extraterrestrial samples preserve signatures of a planetesimal's evolutionary history. In particular, samples from smaller bodies provide insight into processes, such as core-formation and magma ocean crystallization, that have been overprinted on Earth. The meteorites that are important for planetary evolution include partially differentiated and fully differentiated samples of irons, stony achondrites and stony-irons. Studies of these samples include whole rock analyses of sample aliquots, in situ measurements of polished mounts, and experimental studies that simulate conditions of parent body formation and evolution. Complementary studies include observational measurements of planetary analogs on Earth, and modeling studies of solar system planetary bodies and exoplanets.

\section{Key Research Areas in Planetary Evolution}

\section{Metal-Silicate Differentiation}

During the early melting of a planetesimal, metal and silicate phases begin to separate from each other. If the planetesimal retains sufficient heat for metal and silicate melting, this process leads to the formation of a core. The conditions and signatures of early metal-silicate differentiation are difficult to ascertain on fully formed planets, particularly those that have experienced later tectonic and impact resurfacing. Ancient meteorites that derive from partially and fully differentiated parent bodies are therefore important for ascertaining the conditions and processes of early metal-silicate differentiation and core-formation on planetary bodies in the solar system.

Our understanding of metal-silicate differentiation primarily comes from measurements of achondrite meteorites and related experimental samples. These studies typically provide constraints using siderophile (iron-loving) and highly siderophile elements (e.g., Kleine et al., 2009; Day et al., 2016 and references therein, Chabot et al., 2017), but also investigate the metal partitioning behavior of light elements (e.g., Fischer et al. 2015). Quantifying the sinks of volatile 
elements into metal-phases during core-formation can be important for assessing planet-scale volatile inventories, which have implications for habitability on rocky planetary bodies (e.g., Hirschmann, 2016).

This work lends insight into the range of conditions of metal-silicate differentiation and coreformation that occurred on terrestrial planetesimals and Earth. This knowledge can be readily applied to interpreting remote-sensing data, both from missions to primitive asteroids within the solar system (e.g., Psyche), as well as those that observe exoplanets farther afield (e.g., Schaefer et al., 2017). The constraints from these studies are further valuable for determining the cooccurrence of core-formation and a persisting magnetic field, which could be an important requisite for habitability on Earth-like exoplanets (e.g., Lazio et al., 2019).

\section{Scientific Questions:}

1. What is the full range of metal-silicate differentiation conditions represented in our meteorite collections?

2. How do distinct sets of conditions affect light element element partitioning between the core and mantle of a planetary body?

\section{Recommendations:}

- Prioritize observational and experimental geochemical studies of meteorites and analogs that will investigate the range of temperatures, pressures and oxygen fugacity conditions of core-formation for solar system planetary bodies.

- Emphasize the need to quantify metal-silicate partitioning behavior and the resulting metal and silicate compositions, for the full range of scenarios represented by meteorites and their parent bodies.

- Support observational and experimental studies that investigate the links between coreformation and magnetism during planetary evolution, as well as complementary remotesensing and modeling efforts with attention to exoplanets.

\section{Silicate Melting}

The pathways of silicate melting and differentiation on planetary bodies are important determinants of subsequent volcanism styles, as well as the retention (or loss) of volatile elements. Large-scale magma oceans and localized silicate melting broadly represent two end-members of silicate differentiation. The constraints on silicate melting from meteorites are important both for asteroids and in scenarios where smaller, differentiated (and possible volatile depleted) planetesimals accreted to form larger planetary bodies.

Our understanding of the magma ocean stage in planetary differentiation, and subsequent crystallization, is largely based on geochemical studies of lunar samples from the Apollo missions (e.g., Elardo et al., 2011 and references therein). Applying that framework to other meteorite groups, and their respective parent bodies, has demonstrated that magma oceans are likely widespread during the evolution of planets and planetesimals (e.g., Greenwood, 2005; 2017; Schaefer and Elkins-Tanton, 2018). 
Although magma oceans seem to have occurred on a number of planetary bodies in the solar system, planets with insufficient heating sources or mass to maintain silicate melting temperatures were unlikely to have experienced a global magma ocean (e.g., Neumann et al., 2014). In these instances, mantle differentiation likely proceeded through heterogeneous and localized silicate melting, leading to distinct mantle domains within a single parent body. This mantle heterogeneity may be observed in the compositions of primitive volcanic meteorites, as in the case of howarditeeucrite-diogenite parent body (e.g., Lunning et al., 2015). Further investigation of evidence for localized silicate melting on meteorite parent bodes is important for identifying the critical thresholds (e.g., mass, temperature, pressure, internal heating sources) below which planetesimals cannot sustain global-scale melting (e.g., Elkins-Tanton, 2012 and references therein).

For planets that can sustain global-scale melting, magma oceans are important in generating thick steam atmospheres, which help retain volatiles (e.g., Hier-Majumder and Hirschmann, 2017). At the same time, magma oceans also facilitate outgassing, which can lead to atmospheric loss (e.g., Tucker and Mukhopadhyay, 2014). For smaller planetesimals, or those with insufficient radioactive heating elements, silicate melting in the absence of an atmosphere may have led to the more rapid loss of volatiles.

Linking early silicate differentiation to the mantle reservoirs of subsequent volcanism can help characterize the progression from chondritic precursor material to differentiated achondrites. This is particularly important for ascertaining the compositional range of igneous and volcanic products within the solar system (e.g., Collinet and Grove, 2020), as well as their eruption styles (e.g., Byrne, 2020). In turn, volcanism influences planetary evolution through the release of gases to the atmosphere; the inventories of water, carbon-dioxide and sulfur in a planetary atmosphere have important implications for the presence of liquid surface water, and hence life. Understanding silicate melting, from differentiation to volcanism, is important for identifying typical planetary evolutionary trajectories for potentially habitable exoplanets (e.g., Kite et al., 2009).

\section{Scientific Questions:}

1. What are the conditions on young planetary bodies that lead to large-scale magma oceans, compared to those that only allow for localized silicate melting?

2. What is the role of precursor material and silicate differentiation processes in determining the types of volcanism on planetary bodies?

\section{Recommendations:}

- Prioritize observational studies of meteorites that would provide constraints on planetary body scenarios that lead to magma ocean differentiation compared to localized silicate differentiation.

- Emphasize the need to better understand the styles of volcanism on solar system bodies, both from meteorites and remote sensing (e.g., Io), to determine constraints for the observations of volcanism on exoplanets.

- Support observational, experimental and modeling approaches to understanding element partitioning during silicate differentiation on planets with unique precursor materials, with the aim of ascertaining the types of volcanism possible on these worlds. 


\section{$\underline{\text { Impact Bombardment }}$}

Impact bombardment is considered an important process in the history of this solar system, and it occurs on planetary bodies throughout their evolution. Examples include the break-up of asteroid bodies, Earth's moon-forming giant impact and late-stage volatile delivery by impactors to the terrestrial planets.

The signatures of impact bombardment prior to core-formation are overprinted by element partitioning during metal-silicate differentiation, as siderophile elements are sequestered into the iron core. During late-accretion, in which impacts occur after core-formation, siderophile elements typically can no longer partition into the core and hence remain in mantle materials (c.f., Marchi et al., 2018). Evidence of late-accretion is reflected in elevated absolute abundances of siderophile elements in mantle materials, higher than expected for metal-silicate differentiation (e.g., Day et al., 2016 and references therein). There is evidence of late-accretion for a number of planetary bodies, including the Earth and Moon, that has provided constraints for the contribution of these late impactors to planets (Walker, 2009; Bottke et al., 2010).

Studies of meteorites, as well as terrestrial samples, indicate that rocky planetesimals experienced significant volatile depletion when compared to precursor materials (Day and Moynier, 2014 and references therein). Although impact erosion is considered to have a role in the volatile depletion of rocky bodies (e.g., Genda and Abe, 2003; 2005; Schlichting and Mukhopadhyay, 2018), differences in planetary mass, outgassing and atmospheric pressure can also influence volatile inventories. The case of the present-day Earth, with surface conditions that allow for liquid water, suggests the possible role of late-accretion impactors in delivering and mobilizing volatiles on the Earth (e.g., Albarede et al., 2013; Marchi et al., 2016). Over Earth's history, the timing, amount and composition of impact bombardment, including associated erosion events and delivery of volatiles, may have influenced the ultimate habitability of the planet.

As a nearly ubiquitous process in our solar system, understanding the full range of impact bombardment styles and effects within our solar system will have important implications for the habitability of exoplanets, particularly those that are close to their host star and have experienced significant volatile depletion. Next-generation imaging capabilities may provide the capability to identify physical evidence of impact bombardment on extra-solar planetary bodies, providing constraints on the late-accretion flux within distant solar systems. In order to best interpret this data, we will need to increase our understanding of the role of impact bombardment on habitability within our own solar system.

\section{Scientific Question:}

1. What are the major types of impactors and timings of bombardment events during planetary evolution that affect a planet's habitability?

2. How does a planet's impact bombardment history affect episodes of volatile delivery and loss over its evolution?

\section{Recommendations:}


- Prioritize observational studies of meteorites that provide constraints on the timing and extent of impact bombardment on their parent bodies, with attention to evidence of volatile delivery and/or loss.

- Emphasize the need to better understand the role of impact bombardment on the unique evolutionary trajectories of the terrestrial planets and planetsimals in this solar system and apply this knowledge to exoplanet modeling and observations.

- Support complementary observational, experimental and modeling efforts on Earth's impact bombardment history, particularly where study findings have important implications for habitability.

\section{References}

1. Albarede, F., Ballhaus, C., Blichert-Toft, J., Lee, C. T., Marty, B., Moynier, F., \& Yin, Q. Z. (2013). Asteroidal impacts and the origin of terrestrial and lunar volatiles. Icarus, 222, 44-52.

2. Chabot, N. L., Wollack, E. A., McDonough, W. F., Ash, R. D., \& Saslow, S. A. (2017). Experimental determination of partitioning in the Fe-Ni system for applications to modeling meteoritic metals. Meteoritics \& planetary science, 52, 1133-1145.

3. Day, J. M. D., \& Moynier, F. (2014). Evaporative fractionation of volatile stable isotopes and their bearing on the origin of the Moon. Philosophical Transactions of the Royal Society A: Mathematical, Physical and Engineering Sciences, 372, 20130259.

4. Day, J. M. D., Brandon, A. D., \& Walker, R. J. (2016). Highly siderophile elements in Earth, Mars, the Moon, and asteroids. Reviews in Mineralogy and Geochemistry, 81, 161238.

5. Elkins-Tanton, L. T. (2012). Magma oceans in the inner solar system. Annual Review of Earth and Planetary Sciences, 40, 113-139.

6. Fischer, R. A., Nakajima, Y., Campbell, A. J., Frost, D. J., Harries, D., Langenhorst, F., Miyajima, N., Pollok, K. \& Rubie, D. C. (2015). High pressure metal-silicate partitioning of Ni, Co, V, Cr, Si, and O. Geochimica et Cosmochimica Acta, 167, 177194.

7. Genda, H., \& Abe, Y. (2003). Survival of a proto-atmosphere through the stage of giant impacts: the mechanical aspects. Icarus, 164, 149-162.

8. Genda, H., \& Abe, Y. (2005). Enhanced atmospheric loss on protoplanets at the giant impact phase in the presence of oceans. Nature, 433, 842-844.

9. Greenwood, R. C., Burbine, T. H., Miller, M. F., \& Franchi, I. A. (2017). Melting and differentiation of early-formed asteroids: The perspective from high precision oxygen isotope studies. Geochemistry, 77, 1-43.

10. Greenwood, R. C., Franchi, I. A., Jambon, A., \& Buchanan, P. C. (2005). Widespread magma oceans on asteroidal bodies in the early solar system. Nature, 435, 916-918.

11. Hier-Majumder, S., \& Hirschmann, M. M. (2017). The origin of volatiles in the E arth's mantle. Geochemistry, Geophysics, Geosystems, 18, 3078-3092.

12. Schaefer, L., \& Elkins-Tanton, L. T. (2018). Magma oceans as a critical stage in the tectonic development of rocky planets. Philosophical Transactions of the Royal Society A: Mathematical, Physical and Engineering Sciences, 376, 20180109.

13. Schlichting, H. E., \& Mukhopadhyay, S. (2018). Atmosphere impact losses. Space Science Reviews, 214, 34. 
14. Walker, R. J. (2009). Highly siderophile elements in the Earth, Moon and Mars: update and implications for planetary accretion and differentiation. Geochemistry, 69, 101-125.

15. Schaefer, L., Jacobsen, S. B., Remo, J. L., Petaev, M. I., \& Sasselov, D. D. (2017). Metal-silicate partitioning and its role in core formation and composition on super-Earths. The Astrophysical Journal, 835, 234.

16. Lazio, J., Hallinan, G., Airapetian, A., Brain, D. A., Clarke, T. E., Dolch, T., Dong, C.F., Driscoll, P.E., Fares, R., Griessmeier, J.M. \& Farrell, W. M. (2019). Magnetic Fields of Extrasolar Planets: Planetary Interiors and Habitability. Bulletin of the American Astronomical Society, 51, 135.

17. Elardo, S. M., Draper, D. S., \& Shearer Jr, C. K. (2011). Lunar Magma Ocean crystallization revisited: Bulk composition, early cumulate mineralogy, and the source regions of the highlands Mg-suite. Geochimica et Cosmochimica Acta, 75, 3024-3045.

18. Neumann, W., Breuer, D., \& Spohn, T. (2014). Differentiation of Vesta: Implications for a shallow magma ocean. Earth and Planetary Science Letters, 395, 267-280.

19. Collinet, M., \& Grove, T. L. (2020). Widespread production of silica-and alkali-rich melts at the onset of planetesimal melting. Geochimica et Cosmochimica Acta, 277, 334357.

20. Byrne, P. K. (2019). A comparison of inner Solar System volcanism. Nature Astronomy, 1-7.

21. Kite, E. S., Manga, M., \& Gaidos, E. (2009). Geodynamics and rate of volcanism on massive Earth-like planets. The Astrophysical Journal, 700, 1732.

22. Hirschmann, M. M. (2016). Constraints on the early delivery and fractionation of Earth's major volatiles from $\mathrm{C} / \mathrm{H}, \mathrm{C} / \mathrm{N}$, and $\mathrm{C} / \mathrm{S}$ ratios. American Mineralogist, 101, 540-553.

23. Marchi, S., Black, B. A., Elkins-Tanton, L. T., \& Bottke, W. F. (2016). Massive impactinduced release of carbon and sulfur gases in the early Earth's atmosphere. Earth and Planetary Science Letters, 449, 96-104.

24. Kleine, T., Touboul, M., Bourdon, B., Nimmo, F., Mezger, K., Palme, H., Jacobsen, S.B., Yin, Q.Z. \& Halliday, A. N. (2009). Hf-W chronology of the accretion and early evolution of asteroids and terrestrial planets. Geochimica et Cosmochimica Acta, 73, 5150-5188.

25. Bottke, W. F., Walker, R. J., Day, J. M.D., Nesvorny, D., \& Elkins-Tanton, L. (2010). Stochastic late accretion to Earth, the Moon, and Mars. science, 330, 1527-1530.

26. Marchi, S., Canup, R. M., \& Walker, R. J. (2018). Heterogeneous delivery of silicate and metal to the Earth by large planetesimals. Nature geoscience, 11, 77-81. 